\title{
A HYBRID POINT CLOUD REALITY CAPTURE FROM TERRESTRIAL LASER SCANNING AND UAV-PHOTOGRAMMETRY
}

\author{
Ahmad Firdaus Razali ${ }^{1}$, Mohd Farid Mohd Ariff ${ }^{2}$, Zulkepli Majid ${ }^{3}$ \\ Geospatial Information and Imaging Research Group (GI2RG), \\ Faculty of Built Environment \& Surveying, Universiti Teknologi Malaysia, Johor Bahru, MALAYSIA \\ afirdaus65@graduate.utm.my1,.mfaridma@utm.my², zulkeplimajid@utm.my³
}

\section{Commission II}

KEY WORDS: Terrestrial laser scanning, photogrammetry, point clouds, geospatial mapping

\begin{abstract}
:
Point clouds are a digital representation of physical objects or buildings that exist in real world. There are many sources that a point cloud can come from such as a terrestrial laser scanner (TLS) or an unmanned aerial vehicle (UAV). This paper presents a simple method of integrating point clouds from two (2) data sources; TLS and UAV using simple alignment of rigid body transformation method known as Point Pair Picking (PPP). The point cloud data are the representation of details of a one-story building located in Johor Bahru, Malaysia. The process of aligning two (2) separate clouds into one (1) dataset requires initial processing such as noise removal before the alignment process was started. A laser (LAS) formatted data were formed so that it compatible with the PPP process. As the result, a high dense hybrid cloud-model was produced covering complete details of the building. This shows that integration of point clouds could improve 3D documentation assessment such as Building Information Modelling (BIM) by contributing richer semantic information.
\end{abstract}

\section{INTRODUCTION}

Point clouds are a digital representation of physical objects or buildings that exist in real world. Point clouds provide as-built features and geospatial information of the represented details such as as-is red-green-blue (RGB) colours, dimensional measurement, size and volumetric property. Point clouds are generated by powerful lens which capable to record the objects (Saptari, Hendriatiningsih, Bagaskara, \& Apriani, 2019) through Light Detection and Ranging system (LiDAR) which usually installed in a geodetic clouds instrument known as terrestrial laser scanner (TLS). Other than that, point clouds also can be generated from cheaper (Sharif, Hazumi, \& Meli, 2018; Zięba, 2016) and simpler platform compared to TLS but using imaging system known as UAV-photogrammetry.

This paper presents the methods of incorporating two different point clouds data source; TLS and UAV becoming one (1) complete cloud model known as hybrid point clouds. Cloud registration is the process of generating hybrid point clouds.

There are three (3) types of cloud registration method that are commonly applied in the industry namely, Iterative Closest Points (ICP) (Li, Wang, Wang, \& Tao, 2020), Feature Extraction and Matching (FEM) (Liu, Kong, Zhao, Gong, \& Han, 2018) and Match Box Bounding Centre (MBBC) (Ahmad Fuad, Yusoff, Ismail, \& Majid, 2018).

\section{REALITY CAPTURE - GEODETIC TOOLS}

\subsection{Terrestrial Laser Scanner}

Terrestrial laser scanner (TLS) is a land surveying tool which captures topography and details on the ground. It works as a terrestrial version of a LiDAR system where point clouds are generated from the scanning process covering $180^{\circ}$ and $270^{\circ}$ direction for horizontal and vertical captures respectively.

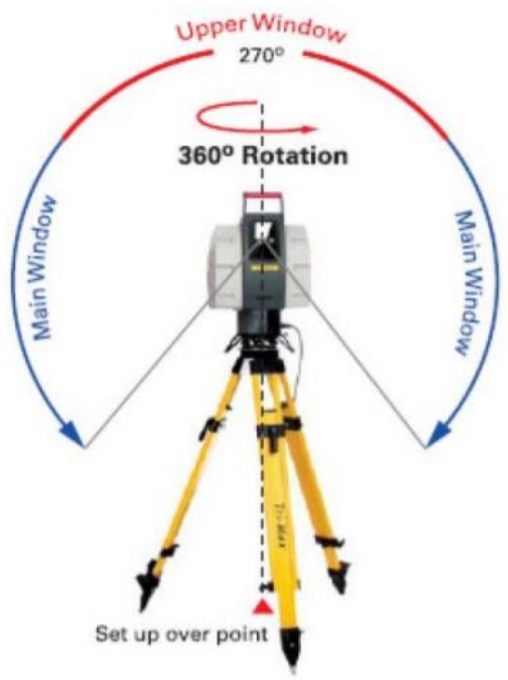

Figure 1. TLS Scanning direction (Cătălina \& AndreeaFlorina)

The capability of performing accurate measurement, efficient and able to produce millions of point clouds (Stober, Žarnić, Penava, Podmanicki, \& Virgej-Đurašević, 2018) makes this tool is better than conventional land surveying tool such as GNSS and total station. The advantage of TLS is that it records $3 \mathrm{D}$ geospatial data compared to GNSS and total station which limit to point- based data acquisition (Razali et al., 2020). TLS measures distance from a fixed position within the target area by transmitting laser pulses to the target. Millions of points that contain 3D coordinates are derived during the laser transmission or scanning process. These points are called point clouds. Point clouds 
spatially represents geometry and as-built visualisation of the scanned object.

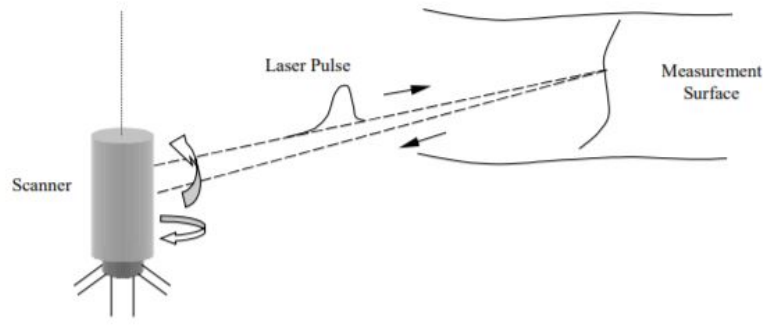

Figure 2. TLS principle (Lichti, Stewart, Tsakiri, \& Snow, 2000)

\subsection{UAV-Photogrammetry}

Unmanned aerial vehicle (UAV) or commonly known as a drone is an advanced imaging technology nowadays. UAV is used as a platform for photogrammetry surveying as an alternative for expensive airborne photogrammetry. Instead, UAV is much preferable especially when involves small area which airborne mapping is not worth it. For geomatics aspect, UAVphotogrammetry is an image-based surveying technique where topographic properties such as area, volume, digital surface model (DSM), digital terrain model (DTM) and digital elevation model (DEM) are extracted. Figure 3 below shows the principle of UAV- photogrammetry mapping. The flight path is designed for image overlapping during data acquisition.

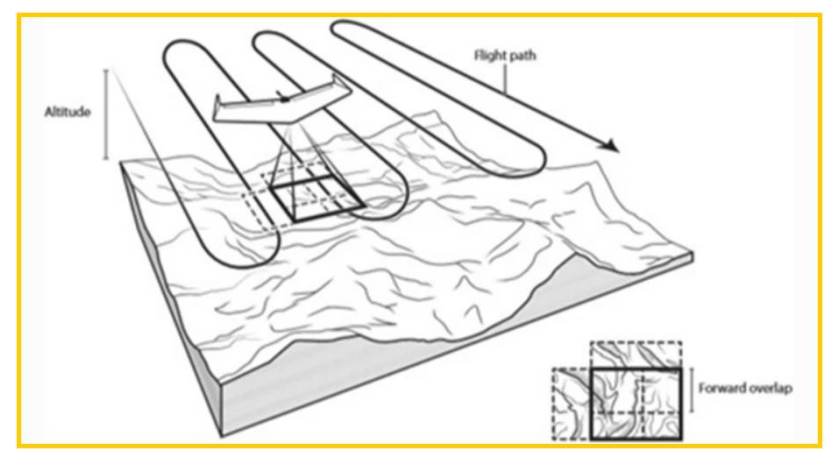

Figure 3. UAV-photogrammetry mapping (F, 2011)

For practicality aspect, UAV-photogrammetry can be utilised for mountainous (Karachaliou, Georgiou, Psaltis, \& Stylianidis, 2019) and high planar area (Jo \& Hong, 2019). This technique is also suitable for non-topographic purpose such buildings data acquisition rooftop details are able to be captured which TLS and other geodetic tools are unable to support. Looking at the limitations of coverage area, Croce, Caroti, Piemonte, and Bevilacqua (2019) suggest that 3D reconstruction of architectural details by combining with other geodetic tools is applicable. Additionally, with its advantage of low cost surveying technique (Sharif et al., 2018; Zięba, 2016), UAV-photogrammetry has potential for infrastructure decision-making such as transportation management (Escobar-Wolf, Oommen, Brooks, Dobson, \& Ahlborn, 2018). For cost effectiveness aspect, Ahmet and Yakar (2018) agree that this technique is safe, fast and accurate for detailing works.

\section{REGISTRATION METHOD}

\subsection{Iterative Closest Point (ICP)}

ICP is the technique of aligning two entities of point cloud datasets. This process is called rigid body transformation where matrix T-translation and R-rotation are defined. According to Fujimoto, Kimura, Beniyama, Moriya, and Nakayama (2009), ICP is the process of geometrical alignment with initial approximation of relative pose is known. They added, the two sets of data used are iteratively transformed which point pairing on the surface is created and error metric is minimised. This was also supported by Li et al. (2020) where they emphasised that ICP runs iterative rigid body transformation between two sets of data.
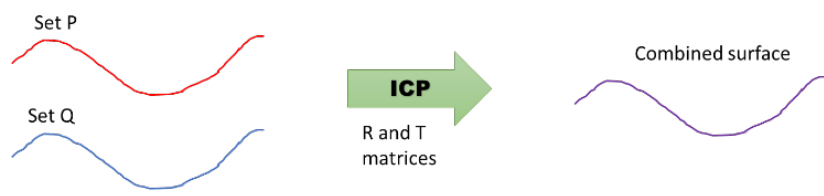

Figure 4. Concept of ICP

Equation 1 shows the mathematical formula of ICP (Stachniss, 2021).

$$
\sum_{(i, j) \in C}\left\|y_{i}-R x_{j}-t\right\|^{2} \rightarrow \min
$$

where,

$$
\begin{aligned}
& \mathrm{Y}=\text { Point cloud (source) } \\
& \mathrm{X}=\text { Point cloud (target) } \\
& \mathrm{R}=\text { Rotation matrix } \\
& \mathrm{T}=\text { Translation vector }
\end{aligned}
$$

Assuming coordinates of $\mathrm{P}$ and $\mathrm{Q}$ :

$$
\begin{aligned}
& \mathrm{Y}=\left\{y_{i} \mid 1=1,2,3 \ldots\right\} \\
& \mathrm{X}=\left\{x_{i} \mid 1=1,2,3 \ldots\right\}
\end{aligned}
$$

with correspondences $C=\{(i, j)\}$

\subsection{Feature Extraction and Matching (FEM)}

FEM is an improvised method for point cloud registration. According to Liu et al. (2018), the mathematical model applies point match searching on the point clouds regions. Also, time spent for registration process is minimised without reducingpoint pairing accuracy and the algorithm speed is improved (Liu et al., 2018).

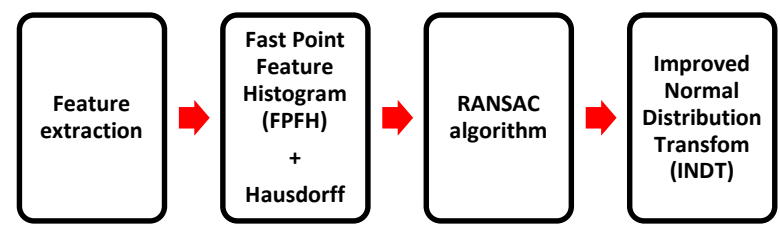

Figure 5. FEM processing phases 
This method applies Equation 2 (Liu et al., 2018) to determine concave and convex point from regions body of point clouds.

Let a point cloud dataset, $\mathrm{P}$;

$$
S(p)=\frac{1}{2}-\frac{1}{\pi} \arctan \frac{k_{1}(p)+k_{2}(p)}{k_{1}(p)-k_{2}(p)}
$$

$p_{i}$ is a local convex point :

$$
S\left(p_{i}\right) \max \left(S\left(p_{i 1}\right), S\left(p_{i 2}\right), \ldots, S\left(p_{i k}\right)\right)
$$

$p_{i}$ is a local concave point:

$$
S\left(p_{i}\right) \min \left(S\left(p_{i 1}\right), S\left(p_{i 2}\right), \ldots, S\left(p_{i k}\right)\right)
$$

$S\left(p_{i 1}\right), S\left(p_{i 2}\right), \ldots, S\left(p_{i k}\right)$

$=S(p)$ value of the point $p_{i}$ neighbourhood points.

The dataset $P$ are extracted by calculating the mean curvature of each point of the dataset. Mean $\mu$ and variance $\sigma$ of the mean curvature are then computed. Feature points are then extracted when concave points are judged by the reserved points.

\subsection{Point Pair Picking (PPP)}

The PPP approach is used to detect common properties between two entities by manually selecting azt least three points in common. The more common spots chosen, the more similar the model will be. In comparison to ICP, PPP is the more robust technique for registering two sets of point clouds since it uses similar features checked by humans, whereas ICP automatically relies on inconsistent surface provided by both datasets. For example, if the first dataset is missing some surfaces/points, ICP is unable to detect its common entities to match with the second dataset identically. As a result, the two datasets may not perfectly overlap.

\subsection{Match Bounding Box Center (MBBC)}

MBBC is the simplest method among the other two methods where two box entities are matched. According to Ahmad Fuad et al. (2018), MBBC requires one reference entity (body of point cloud data) to mapped at the same place with second entity. A transformation matrix derived by MBBC is shown on Figure 6 below while Figure 7 shows the matching process of two cloud bodies.

\begin{tabular}{|l|}
\hline Match Bounding-Box Centers - Computed Transformation Matrix \\
1.0000000000000 .0000000000000 .0000000000000 .281250000000 \\
$0.0000000000001 .0000000000000 .000000000000-0.023437500000$ \\
$0.0000000000000 .0000000000001 .000000000000-2.659999847412$ \\
0.0000000000000 .0000000000000 .0000000000001 .000000000000 \\
\hline
\end{tabular}

Figure 6. MBBC 4x4 matrix (Ahmad Fuad et al., 2018)

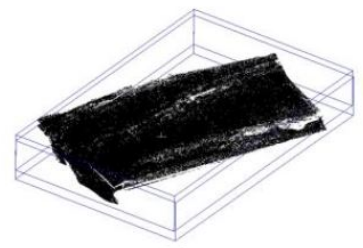

(a)

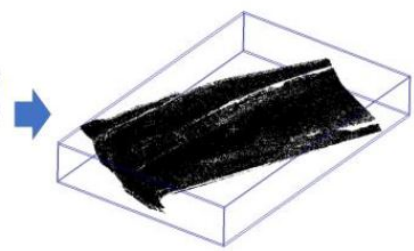

Figure 7. MBBC process (Ahmad Fuad et al., 2018)

\section{THE FORMATION OF HYBRID POINT CLOUDS}

The methodology of this project begins with some literature reviews, developing understanding on the basic and obtaining some idea about the recent projects. Next, data acquisition was carried out using TLS and UAV covering interior and exterior details of a one-storey building and rooftop area respectively.

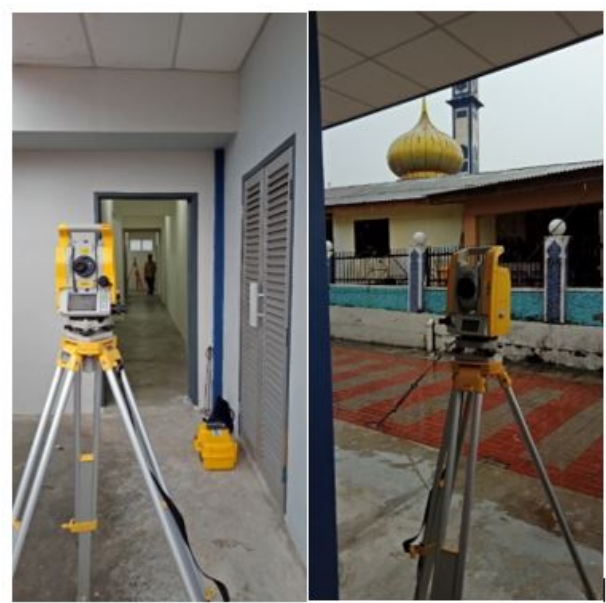

Figure 8. TLS setup

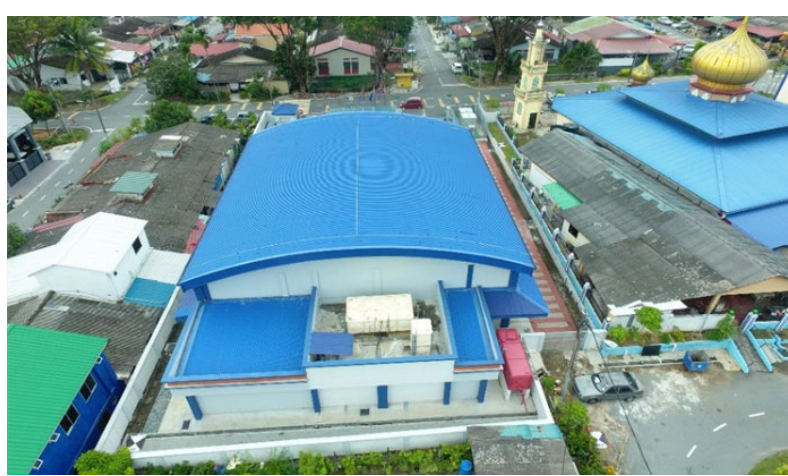

Figure 9. Rooftop area covered by UAV

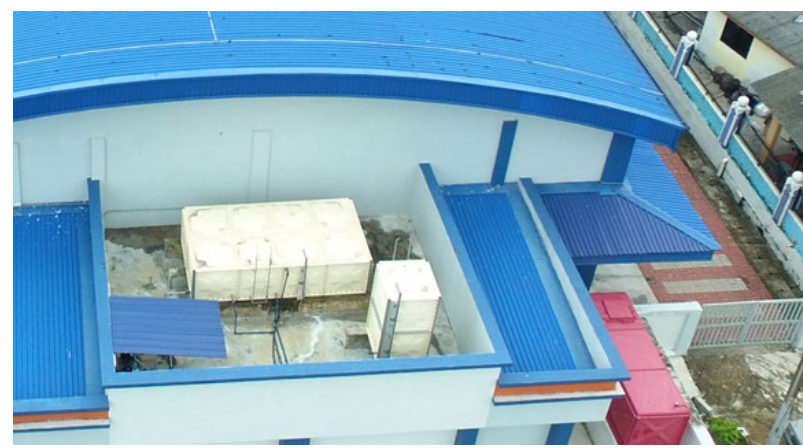

Figure 10. Inaccessible area for TLS which UAV can cover 
Next, data processing involving filtering, noise removal and georeferencing were done using Topcon Scan Master software reduce the density and size of the data. Figure 11 shows flowchart of point cloud processing.

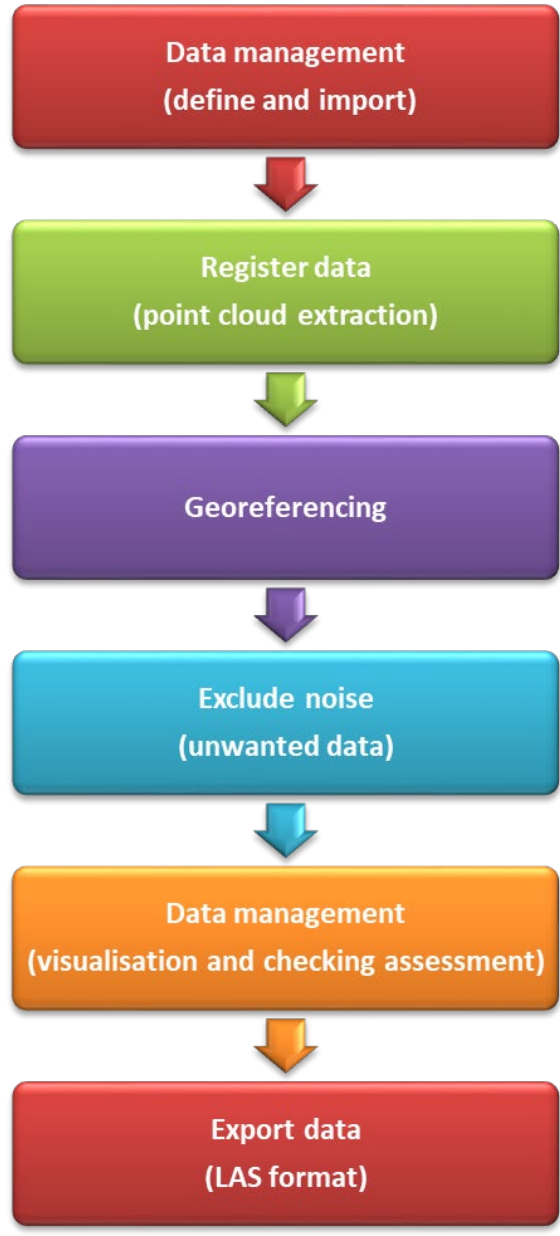

Figure 11. Point cloud processing

Next, point cloud integration was done using CloudCompare software. The method used before a hybrid cloud was formed was the Point Pair Picking (PPP) method because it is more practical compared to other method. Plus, the matching process is not depending on computer interpretation which might cause inconsistent matching. For example, identical entities between the TLS and UAV data might not be exactly interpreted by the software. Hence, it requires human vision to decide which points are the same. However, PPP might be friendly for one (1) pair dataset (e.g. TLS + UAV) because it is manageable, but it might not be practical for many sets of data. Hence, automatic methods such as ICP or MBBC are preferable, but the point clouds must be optimally cleaned from noises so that it will not affect the pairing process.

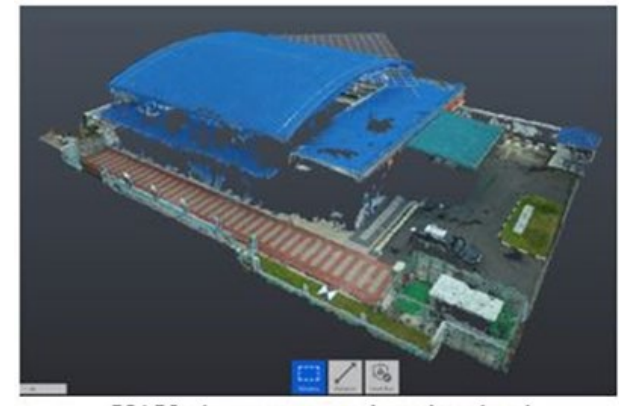

UAV-photogrammetric point cloud

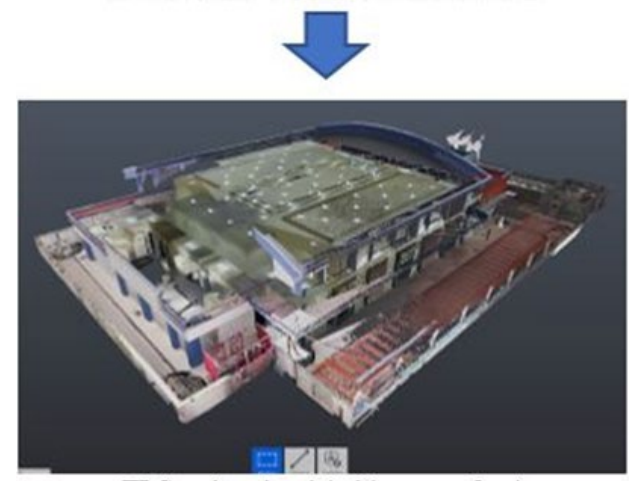

TLS point cloud (without rooftop)

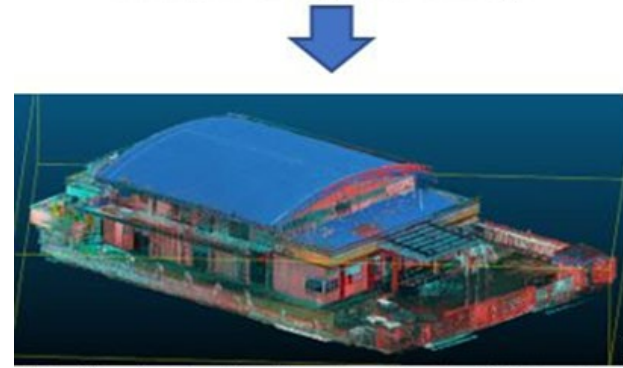

Hybrid point clouds after aligned using PPP method

Figure 12. Hybrid point clouds formation

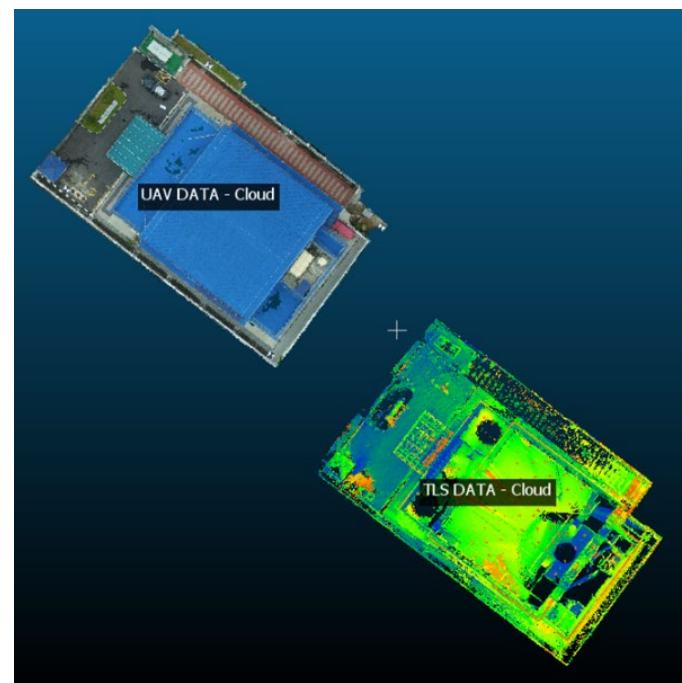

Figure 13. UAV and TLS point cloud (top view)

\section{CONCLUSIONS}

The output of this project is a hybrid cloud model which is completely representing the whole area of the building including rooftop, interior and exterior of the building. The disadvantage of 
hybrid clouds is that it limits to geometry without semantic value. Meanwhile, the advantage of hybrid clouds is that they have asbuilt visualisation and geometry which smoothing 3D modelling assessment. However, the rendering operation in 3D modelling reduces geometric measurement, but it has semantic information.

\section{ACKNOWLEDGEMENT}

The authors highly acknowledge to Universiti Teknologi Malaysia for supporting this study under research grant Vot No. $21 \mathrm{H} 09,05 \mathrm{G} 12$. The research has been financially supported by Universiti Teknologi Malaysia under Research Grant Scheme UTM Fundamental Research Proposal (QJ120000.2552.21H09) and the ministry of Higher Education of Malaysia under Fundamental Research Grant Scheme 2021 (RJI 30000.7852.5F448).

\section{REFERENCES}

Ahmad Fuad, N., Yusoff, A., Ismail, Z., \& Majid, Z. (2018). Comparing the performance of point cloud registration methods for landslide monitoring using mobile laser scanning data. International Archives of the Photogrammetry, Remote Sensing and Spatial Information Sciences, 42(4/W9), 11-21.

Ahmet, Ş., \& Yakar, M. (2018). Photogrammetric modelling of hasbey dar'ülhuffaz (masjid) using an unmanned aerial vehicle. International Journal of Engineering and Geosciences, 3(1), 6-11.

Cătălina, C., \& Andreea-Florina, J. The use of Terrestrial Laser Scanning in GIS applications

Croce, V., Caroti, G., Piemonte, A., \& Bevilacqua, M. G. (2019). Geomatics for Cultural Heritage conservation: integrated survey and $3 D$ modeling. Paper presented at the 2019 IMEKO TC-4 International Conference on Metrology for Archaeology and Cultural Heritage.

Escobar-Wolf, R., Oommen, T., Brooks, C. N., Dobson, R. J., \& Ahlborn, T. M. (2018). Unmanned aerial vehicle (UAV)-based assessment of concrete bridge deck delamination using thermal and visible camera sensors: A preliminary analysis. Research in Nondestructive Evaluation, 29(4), 183-198

F, G. (2011). Drone and aerial observations. In. journalofbigdata.springeropen.com.

Fujimoto, K., Kimura, N., Beniyama, F., Moriya, T., \& Nakayama, Y. (2009). Geometric alignment for large point cloud pairs using sparse overlap areas. Proceedings of SPIE - The International Society for Optical Engineering. doi:10.1117/12.805765

Jo, Y. H., \& Hong, S. (2019). Three-dimensional digital documentation of cultural heritage site based on the convergence of terrestrial laser scanning and unmanned aerial vehicle photogrammetry. ISPRS International Journal of Geo-Information, 8(2), 53.

Karachaliou, E., Georgiou, E., Psaltis, D., \& Stylianidis, E. (2019). UAV for mapping historic buildings: from 3D modelling to BIM. Int. Arch. Photogramm. Remote Sens. Spat. Inf. Sci, 397-402.

Li, P., Wang, R., Wang, Y., \& Tao, W. (2020). Evaluation of the ICP Algorithm in 3D Point Cloud Registration. IEEE Access, $\quad 8, \quad$ 68030-68048. doi:10.1109/ACCESS.2020.2986470

Lichti, D., Stewart, M., Tsakiri, M., \& Snow, A. (2000). Calibration and testing of a terrestrial laser scanner.
International Archives of Photogrammetry and Remote Sensing, 33(B5/2; PART 5), 485-492.

Liu, Y., Kong, D., Zhao, D., Gong, X., \& Han, G. (2018). A Point Cloud Registration Algorithm Based on Feature Extraction and Matching. Mathematical Problems in Engineering, 2018.

Razali, A. F., Ariff, M. F. M., Majid, Z., Darwin, N., Aspuri, A., \& Salleh, M. F. M. (2020, 9-9 Nov. 2020). ThreeDimensional (3D) As-Built Reconstruction from Laser Scanning Dataset. Paper presented at the 2020 IEEE 10th International Conference on System Engineering and Technology (ICSET).

Saptari, A. Y., Hendriatiningsih, S., Bagaskara, D., \& Apriani, L. (2019). Implementation Of Government Asset Management Using Terrestrial Laser Scanner (Tls) As Part Of Building Information Modelling (BIM). IIUM Engineering Journal, 20(1), 49-69.

Sharif, H., Hazumi, H., \& Meli, R. (2018). 3D documenatation of the petalaindera: digital heritage preservation methods using $3 D$ laser scanner and photogrammetry. Paper presented at the IOP Conference Series: Materials Science and Engineering.

Stachniss, C. (2021). ICP \& Point Cloud Registration - Part 1: Known Data Association \& SVD (Cyrill Stachniss, 2021). Retrieved from https://www.youtube.com/watch?v=dhzLQfDBx2Q\& $\mathrm{t}=772 \mathrm{~s} \& \mathrm{ab}$ channel $=$ CyrillStachniss

Stober, D., Žarnić, R., Penava, D., Podmanicki, M. T., \& VirgejĐurašević, R. (2018). Application of HBIM as a research tool for historical building assessment. Civil Engineering Journal, 4(7), 1565-1574.

Zięba, A. (2016). Creating 3D models of game assets with the use of photogrammetry and low-cost equipment. 